\title{
Investigar teoría sociológica del Sur y del Norte: la propuesta del abordaje simultáneo
}

\author{
Alejandro Bialakowsky*
}

Perfiles Latinoamericanos, 26(52) | 2018

DOI: $10.18504 / \mathrm{pl} 2652-002-2018$

Recibido: 3 de mayo de 2016

Aceptado: 21 de marzo de 2017

\section{Resumen}

Este ensayo propone una metodología para abordar simultáneamente perspectivas de la teoría sociológica del Sur y del Norte, a través de sus problemas y dimensiones emergentes. Para ello, se pretende evitar los dispositivos de "distanciamiento temporal" entre Sur y Norte, ya sean los que señalan un supuesto "retraso" del Sur respecto del Norte en los estudios de "recepción", ya los que afirman un mero "relativismo cultural" entre las elaboraciones de ambas regiones. Esto posibilita analizar en un mismo plano distintos aportes a la teoría sociológica desde ciertas encrucijadas teóricas y epocales de una modernidad simultánea y desigual.

\begin{abstract}
This essay proposes a methodology for simultaneously approaching sociological theory perspectives from the South and the North, through its problems and its emergent dimensions. To this end, I intend to avoid the devices of "temporal distancing" between South and North, either those that indicate a supposed "delay" of the South with respect to the North in "reception" studies, or those that assert a mere "cultural relativism" between the elaborations of both regions. This makes it possible to analyze, on the same level, different contributions to sociological theory from certain theoretical and epochal crossroads of a simultaneous and uneven modernity.
\end{abstract}

Palabras clave: teoría social, Sur-Norte, América Latina, simultaneidad, asimetrías sociales. Keywords: Social theory, South-North, Latin America, simultaneity, social asymmetries.

* Doctor en Ciencias Sociales por la Universidad de Buenos Aires. Instituto Gino Germani-CONICET, Buenos Aires, Argentina | alejbialakowsk@gmail.com 


\section{Introducción}

E

el contexto de las últimas décadas de la teoría sociológica argentina y latinoamericana, se han desplegado investigaciones de largo alcance. Con mayor o menor fuerza, un subcampo de preguntas e intereses específicos comenzó a ganar legitimidad. La formación en teoría que se vinculaba a la docencia y a los marcos teóricos de las investigaciones de corte empírico se fue transformando debido a la irrupción de indagaciones sobre determinadas facetas de la teoría sociológica. Aunque esto no es nuevo ni para Argentina ni para Latinoamérica, este momento de fuerte intensidad permitió —y permite — un estudio de los debates teóricos en sociología. Así, las reflexiones de cuño metodológico de la investigación teórica se convirtieron en una preocupación clave. En esa dirección, he propuesto la factibilidad de un "abordaje problemático" (Bialakowsky, 2013) que elude los reduccionismos de las propuestas de las décadas de 1980 y 1990, sin abandonar ni el análisis comparativo y multidimensional de los presupuestos generales de la disciplina ni el lugar central de los estudios de la sociología sobre la modernidad.

Desde la teoría sociológica latinoamericana, parece que nos encontramos frente a una suerte de división internacional del trabajo intelectual. Es cierto que esto atraviesa a todas las relaciones desiguales de producción y circulación de conocimiento de las ciencias sociales entre el "Norte" y el "Sur" — sobre todo Estados Unidos y Europa Occidental, en tanto "centro", y para este trabajo en particular, Latinoamérica, entendida como "periferia"- Relaciones que hoy se encuentran articuladas en la estandarización de los métodos de evaluación, la bibliometría de las revistas de referato internacional, la imposición del inglés como lingua franca, la asimétrica traducción de textos, etc. (Beigel, 2013). Algo aún más patente para la teoría sociológica que parece dividida entre dos ámbitos académicos. Por un lado, desde el centro y sus revistas, instituciones y corrientes, se produciría teoría. Por el otro, se elaborarían desde la periferia recepciones activas de esas teorías —es decir, reinterpretaciones, apropiaciones y aportes críticos- y se utilizarían estas reinterpretaciones en análisis empíricos que se explayan acerca de las singularidades de los espacios sociales del Sur. Considero que es decisivo revisar estas posiciones dado que implican supuestos que legitiman y reproducen formas asimétricas de investigación en teoría. ${ }^{1}$

1 No resulta fácil delimitar la "teoría sociológica latinoamericana" sin recaer en cierto purismo inconducente, es decir, ¿se deben considerar también dentro de ella las reflexiones producidas por latinoamericanos, más allá de dónde se encuentren cuando las producen?; ;y las elaboraciones que tienen entre sus objetos privilegiados a Latinoamérica, sin importar que sus autores sean o no de esta región? Desde el 
Se ha discutido mucho sobre esta cuestión en la literatura y el ensayo latinoamericanos (García, 2010; Schwartz, 2014), por ejemplo, respecto del debate entre nacionalismo y cosmopolitismo; o, desde la historia intelectual e institucional de la sociología (Blanco, 2006; Pereyra, 2010), acerca del proceso de institucionalización de la disciplina. Me interesa retomar estos aportes fundamentales para enfatizar otra línea analítica que permita una metodología que denominaré abordaje simultáneo. Para esto, me detendré en la perspectiva de Fabian (2002) acerca de la negación de la coetaneidad entre dominantes y dominados como forma de reproducción y producción de las relaciones asimétricas entre Sur y Norte, en especial, en cuanto a la elaboración y circulación de conocimiento. Desde allí delinearé una propuesta enfocada en una simultaneidad compleja de problemas en teoría sociológica que no ocluya reflexiones sobre las relaciones jerárquicas entre Norte y Sur, e incluso dentro de esas gigantescas figuras, por ejemplo, entre países o regiones.

De esta manera, a modo de ensayo, propongo una mirada que se aleje de los desfases temporales. Esto permitirá revisar el supuesto "atraso" temporal de la periferia, el cual implica a la mencionada dinámica de las recepciones. Y también sostendrá una crítica al desplazamiento hacia un mero relativismo cultural que, en general, conduce a una relativización o "desprecio" de la teoría en el Sur. Así, esta simultaneidad habilita a recuperar los aportes a la teoría sociológica desde el Sur, sin por ello dejar fuera del análisis a las relaciones que existen entre estos y los del Norte (de dominación, de apropiación, de diálogo, de disputa), lo cual supone debatir las definiciones mismas de aquello que se entiende como Sur y Norte. Este abordaje, por otra parte, implica un estudio, desde sus dimensiones emergentes, de la compleja simultaneidad de perspectivas de la teoría sociológica en una modernidad simultánea y desigual.

\section{La pregunta por la investigación de teoría sociológica en América Latina}

Durante las últimas décadas en América Latina, por ejemplo en México (Girola \& Zabludovsky, 1991; Zabludovsky, 2002), se ha desplegado una intensa

abordaje simultáneo que aquí propongo, resultan claves las diferencias entre el Sur y el Norte en cuanto a la producción de teoría. Por este motivo, me focalizo en perspectivas cuyos autores han tenido su inserción mayoritaria en espacios académicos latinoamericanos. Creo que la dificultad por delimitar tales espacios se explica en gran medida por las relaciones de dominación implicadas en su constitución y de la dispersión por motivos políticos, económicos o académicos de muchos intelectuales latinoamericanos, los cuales quedan en una especie de posición "intersticial”. Unas excelentes reflexiones sobre esto se hallan en el número monográfico dedicado a esta temática de la revista Papeles del CEIC, de marzo de 2015, http://www.ehu.eus/ojs/index.php/papelesCEIC/issue/view/1242

A. Bialakowsky | Investigar teoría sociológica del Sur y del Norte: la propuesta del abordaje simultáneo Perfiles Latinoamericanos, 26(52) | FLACso México | DoI: 10.18504/pl2652-002-2018 
reflexión sobre la actividad teórica en sociología. Para comprender la especificidad de las producciones teóricas latinoamericanas se han releído, en especial, las propuestas de la lógica teórica de Alexander y la metateoría de Ritzer, las cuales habían acompañado el retorno de la gran teoría en sociología que tuvo su epicentro en la década de 1980 (por nombrar algunos de sus referentes: Bourdieu, Giddens, Habermas y Luhmann).

Así, se sostiene una reivindicación de la actividad teórica, en específico a partir de los análisis comparativos. Siguiendo a Alexander (1982), esa actividad implica un análisis multidimensional de presupuestos generales del continuo científico entre los inalcanzables entornos metafísico y empírico: desde los abstractos presupuestos generales hasta las observaciones empíricas con múltiples componentes en el medio. A su vez, desde Ritzer (1990) al trazar un arco entre paradigmas en competencia, singulares pero comparables, se propugna por un estudio metateórico y multiparadigmático de las polaridades de la disciplina. Sin embargo, en las perspectivas de ambos autores, la defensa de un subcampo teórico dentro del proyecto sociológico de análisis de la modernidad no escapa del clásico marco de referencia de Parsons: la sociología se debate entre el individualismo y el colectivismo, y entre el materialismo (u objetivismo) y el idealismo (o subjetivismo) (Requena, 2000).

Por eso, surgieron nuevos estudios acerca de tal actividad de investigación teórica (Aronson, 2011; Cristiano et al., 2015; Girola, 2015; Mascareño, 2008; Pignouli, 2015; Zabludovsky, 2012). Entre ellos, en otro texto (Bialakowsky, 2013), he propuesto la factibilidad de un abordaje problemático que evite los reduccionismos de tales perspectivas, sin que ello signifique desatender los rasgos propios de la actividad teórica. Desde este abordaje se pueden analizar de forma comparativa distintas perspectivas a partir de la emergencia de una serie de preguntas y reflexiones que se interrogan frente a un problema (el sentido, la dominación, la comunidad, las representaciones, las clasificaciones sociales, entre otros). En el contexto de encrucijadas tanto teóricas como epocales que atraviesan a los distintos autores y sus elaboraciones, es posible demarcar dimensiones claves para cierto problema, el cual no está delimitado por un concepto o unas dicotomías clásicas de la disciplina. Así, por ejemplo, para la comunidad se pueden señalar las dimensiones histórica, tipológica, utópica, tecnológicosocial y ontológica (De Marinis, 2012). Se trata de una alternativa que permite tanto el estudio de una obra específica como la comparación de varias miradas desde las dimensiones emergentes en la investigación.

No obstante, esta propuesta aún resulta abstracta y general. Por ende, requiere ser revisada y complementada con una reflexión decisiva sobre las características singulares de la investigación teórica en América Latina, en el marco de las desiguales relaciones entre Sur y Norte. En tal sentido, en sus estudios sobre la 
modernidad y sus formas de saber, la perspectiva decolonial señala los diversos mitos que se vinculan a las modalidades de dominación entre Norte y Sur, en especial respecto de América Latina: la autogeneración y superioridad histórica de la "civilización" y la "cultura" provenientes del Norte (Lander, 2000). A partir de ese contexto, una pregunta por la circulación internacional de las ideas (Bourdieu, 2000) evidencia la distancia jerárquica entre espacios de saber. Esta asimetría no está solo sostenida por el Norte, sino también por el Sur, ya que tal distancia se intersecta con posiciones y usos de los campos académicos para legitimarse "hacia adentro".

De las clasificaciones sociales que recorren el nexo entre Norte y Sur (Quijano, 2014), es fundamental la división entre la teoría (unida a la abstracción, la racionalidad y la universalidad), supuestamente propia de los espacios dominantes, y la práctica (unida a lo concreto, a lo sensible y a lo particular), supuestamente propia de los espacios periféricos (Lander, 1997; Castro-Gómez, 2000). Esta clasificación suele alterar sus atributos positivos y negativos: tanto la teoría como la práctica pueden subordinarse una a la otra, según diferentes juegos y luchas que adoptan las diversas perspectivas de tales espacios sociales.

Así, se ha observado - y se sigue observando- una oscilación entre distintas posiciones (Domingues, 2009). Por una parte, se reivindica el carácter más empírico y concreto de la sociología latinoamericana frente a la abstracción eurocéntrica del Norte, lo cual supone que la teoría es una actividad innecesaria, superflua e incluso sospechosa. Esta tendencia puede inclinarse hacia un relativismo cultural, que esconde los importantes diálogos y lecturas con perspectivas del Norte entramados en la supuesta originalidad local. Asimismo, los aportes teóricos desde Latinoamérica pueden quedar relegados frente a los del Norte, los cuales se recepcionan de forma más o menos creativa en el marco de los análisis empíricos de la sociología latinoamericana.

En esta dirección, el ensayo latinoamericano, sobre todo en su lazo con la literatura y la ciudad letrada (Rama, 1998), se ha interrogado por el lugar y las estrategias posibles para intervenir desde las singularidades latinoamericanas no solo en las propias sociedades sino también en lo universal. Para ello se ha pretendido evitar provincialismos y cosmopolitismos simplificadores, ya sea desde la antropofagia como operación de apropiación y traducción herética y radical, ya sea desde el barroco como sensibilidad en tensión, transfiguradora y combinatoria, así como también desde una interpretación de las complicidades sociales en la profusión e importación de ideas fuera de lugar, que en ese proceso son transformadas (De Campos, 1982; Schwarz, 2014). ${ }^{2}$ En cuanto a

2 Es interesante observar que, por ejemplo, en las carreras universitarias de literatura en Argentina, se establece una división de estilo romántica nacional o regional de las literaturas, con sus obras claves y su 
la perspectiva de las ideas fuera de lugar, García señala que "muestra el potencial crítico universal de las culturas periféricas sin renegar de tal condición en la cultura occidental, sino, justamente, explotándola” (García, 2010: p. 69). En esa línea, por ejemplo, González destaca el "cariz tenuemente argentino" que implica "cierto modo de tomar autores heterogéneos y articularlos entre sî" (González, 2004: p. 227), en la invención que siempre supone un texto respecto de su contexto.

Si bien la cualidad de fuera de lugar de las ideas también se encuentra en el Norte —el liberalismo en Alemania es un ejemplo (Palti, 2014)_, resulta más productivo para la sociología focalizarse en la condición simultánea de elaboración y reflexión sobre sus problemas nodales. ${ }^{3}$ En la actualidad, en el marco más amplio de la teoría social, la teoría sociológica requiere revaluar sus presupuestos tanto de sus dimensiones epistemológicas y metodológicas como de sus análisis sobre la modernidad, al tener en cuenta las críticas y reflexiones que ponen el foco de atención en la producción en el Sur (Boatcă $\&$ Costa, 2010; Scribano, 2004). Así, el abordaje simultáneo propone adoptar una mirada específica sobre la modernidad, a partir de su carácter simultáneo y no autogenerado desde Europa. En esa mirada se inserta una reflexión sobre la teoría sociológica y su metodología de investigación. A partir de allí, se trata de poner en cuestión las formas estereotipadas de comprender las relaciones entre Sur y Norte respecto de la teoría sociológica, al estudiar la reproducción y producción de ciertas formas de asimetría, sin por ello abandonar la ambición de aportar desde el Sur latinoamericano a la teoría sociológica.

\section{La modernidad simultánea y la simultaneidad de las sociologías}

Desde sus comienzos, la sociología tuvo una relación intensa con los viajes (Schrecker, 2012; Offe, 2006). Estos fueron de formación, como los de sociólogos estadounidenses o latinoamericanos a Europa durante el siglo xx; de revelación o de investigación, como los de Weber a Estados Unidos para su

bibliografía secundaria específica: argentina (del siglo xIx, del siglo xx), latinoamericana, francesa, rusa, inglesa, etc. Sin embargo, cuando se abordan temas más teóricos, como la lingüística o la teoría literaria, tienen cierta primacía las perspectivas del Norte sobre las del Sur, con sus creativas recepciones.

3 Se puede afirmar algo semejante acerca de la relación entre ciertas teorías y sus influencias (o recepciones creativas). Cualquier obra de la sociología, producida en el Sur o en el Norte, puede ser interpretada según sus influencias, por ejemplo, aquella decisiva de Kant en Durkheim y Weber o de Parsons en Germani. Pero, lamentablemente, no suele distribuirse de igual modo la relación entre una teoría y sus influencias. Mientras que, en general, a unas teorías (las del Norte) se les otorga entidad propia, al relegar la investigación sobre sus antecedentes a los especialistas, respecto de otras (las del Sur) se enfatiza en sus relecturas, que suelen adscribirlas a una tradición o articulación original de varias de ellas. 
estudio del protestantismo, o vinculados a posteriores institucionalizaciones de la disciplina, como los de Germani desde Italia a la Argentina o de Lazarsfeld desde Austria a Estados Unidos (véase Pereyra, 2010). Tales viajes no se restringieron a ciertos autores singulares, sino que involucraron también permanentes intercambios de miradas y tradiciones. No obstante, al enfatizar en estos viajes, ¿la reflexión no queda atrapada en la cuestión de la recepción, aunque esta se sofistique? Dichos viajes han sido posibles solo por las características simultáneas y la conexión entre historias de la modernidad desigual (Bhambra, 2014).

En esa dirección, desde la antropología, Fabian (2002) reconstruye la negación de la coetaneidad en la observación del Otro como separado de Occidente espacial y temporalmente. A través de distintas marcas textuales, se revelan dos modos de representación sobre el tiempo del Otro, alejado del tiempo presente o universal de un Nosotros occidental: o bien se sitúa al Otro en el tiempo pasado de una única Historia compartida por la humanidad; o bien se lo coloca en un tiempo incomensurable, en un tiempo otro, incluso fuera de la Historia, que no puede compararse con el tiempo occidental. Así, emerge un discurso alocrónico, que reproduce y produce relaciones de dominación y supone dispositivos de distanciamiento temporal entre diferentes prácticas y culturas, plasmados en las formas de observación y escritura que se autodenominan científicas. Allí se revela cómo se entrecruzan los regímenes de historicidad, es decir, las formas propias de cada sociedad de articular y representar el pasado, el presente y el futuro - los modos en que se ordena el tiempo- (Hartog, 2007), con los regimenes de historiográficos, en tanto "presupuestos temporales que subyacen a la escritura histórica” (Mudrovcic, 2013: p. 12). ${ }^{4}$ Según Fabian, esta relación entre el tiempo sociohistórico y la observación escrita del tiempo del Otro se despliega en dos miradas prototípicas: la evolucionista y la relativista.

Por un lado, se evidencia una mirada evolucionista desde la cual, para la antropología, los viajes espaciales hacia otras culturas no occidentales implican un ir hacia el "pasado" de la modernidad. La historia de la humanidad se completa al analizar las sociedades de estadios anteriores al "presente" de Occidente. Esto

4 Hartog (2007) retoma a Koselleck para sugerir la herramienta heurística de los regímenes de historicidad como construcción para la investigación histórica, la cual permite estudios comparativos (y, por ende, el estudio de los modos del tiempo de Otros en la historia). Así, la organización y la definición del tiempo histórico están atravesadas por la tensión entre el espacio de experiencia y el horizonte de expectativas, que se ve intensificada en los momentos de crisis, cuando el tiempo pierde su carácter dado por sentado al ponerse en duda las relaciones entre pasado, presente y futuro. Como seńala Mudrovcic, estos regímenes de historicidad se vinculan a los regímenes historiográficos de la propia escritura del historiador como observador, por dar un caso, en el paso de la historia ejemplar a la historia moderna, para la cual "el futuro esclarece la historia pasada", como en la visión de progreso de la Ilustración (Mudrovcic, 2013: p. 14). 
se aplica de modo inverso a los viajes desde las colonias a la metrópoli, como "un vistazo al futuro”, en muchos casos para la formación de élites políticas, económicas y culturales que posteriormente retornarán a la colonia (Fanon, 2009). ${ }^{5}$

En el marco de la sociología, esto se vincula a las preguntas por la modernización de los países del Sur, que tanta importancia tuvieron en la "fundación" de la sociología en América Latina (Blanco, 2006). A pesar de que al desplegarse teórica y analíticamente no resultaban ni homogéneas ni libres de tensiones y revisiones, las formas más estereotipadas y optimistas de los estudios sobre la modernización de las década de 1950 y 1960 suponían que los modelos, primero europeo y luego estadounidense, señalaban el camino de pasaje hacia una sociedad "plenamente moderna", con sus instituciones y patrones culturales (Eisenstadt, 2013: p. 154). Más allá de la constatación de que esta convergencia hacia el modelo de los países del Norte se ha revelado como fallida, incluso hoy nos encontramos con lineamientos teóricos y políticos que la siguen sosteniendo — por ejemplo, a partir de cierta interpretación homogénea y errónea de la globalización (Eisenstadt, 2000: p. 16).

Sobre esta fallida dinámica del proceso de modernización, en América Latina se desplegó una de las más relevantes contribuciones de sus ciencias sociales y, en especial, de su sociología: la teoría de la dependencia, que, por ejemplo, a posteriori ha sido retomada por la teoría del sistema mundo de Wallerstein (2005). ${ }^{6}$ Para una reflexión sobre la modernidad simultánea, tal perspectiva resulta un antecedente fundamental. Así, a partir de ella, es clave el reconocimiento tanto de las relaciones de dependencia estructural entre Sur y Norte como de sus consecuencias en la formación de las clases sociales y sus prácticas políticas dentro de las sociedades dependientes (por ejemplo, en las dinámicas del colonialismo interno; véase González, 1969). ${ }^{7}$ Es importante destacar que una de las tensiones fundamentales que atravesó a esa corriente se desplegó, justamente, en las discusiones entre las posiciones de corte "internacionalista" y cuño marxista y aquellas de corte nacionalista y popular (Beigel, 2006) —con diferentes mixturas e intentos de combinación.

5 Eisenstadt (2000: p. 14) indica cómo las élites y los intelectuales no europeos - y, después, no estadounidenses- no aceptaban sin más los temas e instituciones de la modernidad occidental. Por el contrario, se apropiaban de ellos de forma selectiva y original.

6 La teoría del sistema mundo de Wallerstein se vincula al denominado giro espacial de la historia (Pascual, 2014). A partir de autores como Soja (1989) y Harvey (1979), el espacio y la territorialidad pasan a entenderse como construcciones históricas de carácter relacional y heterogéneo, que se modifican según los contextos sociohistóricos. Esto obliga a analizar las conexiones entre los diversos espacio-tiempos simultáneos, como desarrollaré más adelante.

7 Respecto de la situación epistémica de la periferia, en tanto parte "inferior" del todo definido por el "centro", Grüner seńala: "desde el (imaginario) Todo sólo se puede ver, justamente, el Todo, mientras que desde la Parte se puede ver la Parte y su (relación conflictiva con) el Todo" (Grüner, 2010: p. 55). 
Por otro lado, también se ha desplegado una perspectiva relativista, que indica unas temporalidades inconmensurables entre distintas sociedades "intraducibles". Esta posición obtura la coetaneidad de tales sociedades, lo cual resulta aún más patente con el olvido de las conexiones temporales implicadas en la modernidad desde los procesos de colonización y las relaciones de dominación instauradas a partir de ellos. Este relativismo cultural no solo adopta una forma posmoderna, sino que ya se observa en las taxonomías culturales del estructuralismo (por ejemplo, en Lévi-Strauss), que niegan las dimensiones procesuales $\mathrm{y}$ temporales de tales sociedades. Aunque este relativismo cultural reivindique las cualidades del Otro de Occidente (incluso recayendo a veces en el mito del buen salvaje), Fabian sostiene que esa negación de la coetaneidad puede contribuir sin proponérselo al "nuevo, vasto, anónimo, pero terriblemente efectivo régimen del colonialismo ausente" (Fabian, 2002: p. 69). Asimismo, es factible decir que, en algunos casos, tal visión queda circunscrita a cierto particularismo fragmentario, reduccionista y simplificador (bajo distintas formas como en el nacionalismo chauvinista).

Por lo tanto, Fabian define a la coetaneidad como "la problemática simultaneidad de diferentes, conflictivas y contradictorias formas de conciencia" (Fabian, 2002: p. 146. Cursivas en el original). Sin detenerme en su definición de la conciencia desde la praxis, considero clave retomar este juego analítico para dar cuenta de la compleja simultaneidad entre posiciones acerca de un problema teórico. Ahora bien, Fabian se pregunta si esta propuesta acerca de la coetaneidad no está influida por las características de la época, signada por aquello que se podría denominar "globalización hegemónica" (Santos, 2003). O, como afirma Birth (2008), ¿no acecha el peligro de caer en un homocronismo de un supuesto tiempo universal, que es el del propio investigador?

A mi entender, en la misma línea analítica de Fabian, ese tiempo "universal" que hoy parece homogeneizarlo todo ha sido una representación del tiempo que o bien, desde los albores de la modernidad colonial, niega la simultaneidad, o bien actualmente la reduce a una mera conexión instantánea, aquello que Hartog (2010) ha llamado presentismo, como nuevo régimen de historicidad en el que impera lo contemporáneo: "lo que se impone es el tiempo real, la simultaneidad de todo con todo y lo continuo" (Hartog, 2014: p. 86). Sin embargo, al cambiar el foco hacia la simultaneidad sociohistórica de los procesos sociales, entre ellos la elaboración teórica en sociología, lejos se está de homogeneizarlos: se revelan sus complejidades, disrupciones y conflictos (por ejemplo, en el abordaje de los múltiples caminos que se abren a partir de cómo se piensa hoy el problema de las clasificaciones en Argentina o en un país europeo). Si esta propuesta no tiene como resultado un tiempo homocrónico, se debe a que la simultaneidad no implica homogenización. Por el contrario, ella supone restituir el tiempo 
negado y comprender las formas de esa negación que se vinculan a relaciones asimétricas, por dar un caso, de producción y circulación de conocimiento. ${ }^{8}$ Las modalidades específicas del tiempo de cada espacio social están atravesadas por la simultaneidad compartida, y muchas veces sufrida, con otros espacios, que puede relevarse en contraste con la temporalidad simultánea presentista y homogeneizadora que se pretende imponer desde ciertas facetas de la denominada globalización. ${ }^{9}$

De esta manera, resultaría productivo revisar críticamente algunos de los principales análisis que sobre la contemporaneidad ha realizado la teoría sociológica. En primer lugar, el de Luhmann (1997) acerca de la sincronización y desincronización entre sistemas funcionales y sus operaciones comunicativas simultáneas de una sociedad mundial. En segundo, el de Giddens (1999) en relación con los mecanismos de desanclaje de una globalización en la que priman las relaciones entre ausentes. Por último, el de Habermas (2000), que busca un cosmopolitismo en una constelación posnacional frente a un mercado capitalista cada vez más internacionalizado.

De esta forma, en su estudio acerca de las posibilidades de una sociología global (Burawoy, 2008), Bhambra $(2010,2014)$ introduce una dimensión fundamental: el carácter conectado de la modernidad y, por ende, también de las sociologías. El abordaje simultáneo se encuentra próximo a esta mirada de conexión, la cual requiere eludir no solo el eurocentrismo explícito de cierta sociología, sino también el implícito, tanto de la propuesta de las múltiples modernidades (Eisenstadt, 2000) —la cual considera que la modernidad comienza

8 No se trata de la simultaneidad de lo no contemporáneo que retoma Koselleck de la célebre frase de Herder para comprender la temporalidad de la modernidad. Palti (2004) critica la fusión de Koselleck, entre la temporalidad del progreso de la Ilustración del siglo xvin, de la cual extrapola su lectura de la modernidad, y la temporalidad de la evolución del romanticismo de Herder del siglo xIX, que supone una temporalidad específica a cada ser según su propia evolución. Es decir, bajo otras figuras, se observan las dos formas de desfase temporal a las cuales aludí antes: el retraso temporal o su inconmensurabilidad.

9 Sobre la relación entre espacios, asimetrías y representaciones, Dubey (2002) analiza las transformaciones de la literatura afroamericana desde 1970. Allí, observa críticamente la reivindicación del Sur estadounidense tradicional, en tanto espacio imaginario comunitario y de formación de la cultura afroamericana. Tal reivindicación imaginaria — que no debe confundirse con la conservadora racista— revela las frustraciones de las migraciones afroamericanas hacia el Norte y su vida urbana. Esta frustración se da en paralelo a las migraciones hacia el Sur a partir de 1970, motivadas por el desarrollo socioeconómico de la región. La autora considera que estas elaboraciones literarias están en consonancia con las "geografías posmodernas y de la diferencia", las cuales se focalizan en el espacio local en detrimento de un análisis temporal (sobre todo frente a una definición eurocéntrica del tiempo universal). Así, enfatizan el carácter situado de la acción política y el conocimiento. No obstante, Dubey señala la falta de estudio de las conexiones entre distintas geografías, lo cual obligaría a una investigación sobre la temporalidad y, en mis términos, de su simultaneidad, ya que en caso contrario: "se vuelve difícil imaginar las relaciones de mediación entre diferentes ámbitos y circunscripciones que resultaría necesario para un cambio social progresivo" (Dubey, 2002: p. 368). 
en Occidente y mantiene su foco allí luego de expandirse de forma múltiple al resto del mundo- como la del llamado a un cosmopolitismo global sin más (Habermas, 2000). Bhambra hace suya la convocatoria de Chakrabarty a provincializar Europa, para así constituir cosmopolitismos provincializados de diversos espacios sociales vinculados a una sociología de las conexiones.

En específico, el abordaje simultáneo que aquí propongo, si bien toma en cuenta esta conexión de las sociologías dentro una modernidad conectada, pretende comparar en un mismo plano teórico perspectivas del Sur y del Norte en una cierta encrucijada epocal. El estudio de las conexiones al modo de una historia intelectual entre estos espacios de producción de teoría — que pueden desplegar los viajes, las lecturas, las recepciones creativas o sus formas institucionales - es un conjunto de investigaciones sumamente útiles para el abordaje simultáneo, pero estas no son su foco principal.

\section{El abordaje simultáneo para la investigación en teoría sociológica}

Las encrucijadas epocales también son encrucijadas teóricas acerca de determinados problemas que se vuelven decisivos, de ciertos conceptos y enfoques que van mutando. Es evidente que en el Sur o en la periferia se ha elaborado teoría, y se la sigue haciendo. Ahora, ¿cómo se la realiza? ¿De qué manera se incorporan sus discusiones a los diálogos más generales de la disciplina? ¿Cómo se analiza lo contemporáneo y la reconstrucción del pasado de esa teoría? A modo de respuestas, otros han enfatizado en algunas de las estrategias del débil, tales como reconocer la autoridad de los dominantes, para dislocarla en sus propios términos; o postularse por fuera del juego, en tanto forma herética de jugarlo.

Aquí propongo otra metodología de investigación que también implica cierta herejía: poner en simultáneo perspectivas del Sur y del Norte para analizarlas a partir de determinados problemas teóricos. Esta simultaneidad en un mismo plano epocal, por ejemplo entre un autor argentino y otro estadounidense, cuestiona la forma convencional de comprender la teoría sociológica. ${ }^{10}$ Esto no implica olvidar los cruces previos entre el Sur y el Norte (las recepciones mutuas, los diá-

10 Aunque se realice un recorte analítico entre diferentes sociologías desplegadas en determinados países (por ejemplo, una comparación sobre un problema teórico entre la sociología argentina y la estadounidense, como el de la estratificación social en las décadas de 1950 y 1960), esto no implica recaer en un "nacionalismo metodológico" (Chernilo, 2007). Como ya he desarrollado, tal recorte presupone las relaciones simultáneas entre Sur y Norte, como así también entre países y dentro de estos, v. gr., se debe tener en cuenta a Latinoamérica como contexto de debate para Argentina, o las disputas entre las escuelas de Chicago y de Harvard en Estados Unidos. Esto no supone disolver ciertas diferencias semánticas que se pueden rastrear entre tradicionales nacionales y regionales (De Marinis, 2013).

A. Bialakowsky | Investigar teoría sociológica del Sur y del Norte: la propuesta del abordaje simultáneo Perfiles Latinoamericanos, 26(52) | FLACso México | DOI: 10.18504/pl2652-002-2018 
logos, los debates en ciernes); por el contrario, se trata de ubicar tales cruces en el mismo nivel y no remitirlos a la unidireccional relación que va desde el Norte hacia el Sur. De igual modo es necesario realizar esto entre perspectivas del Sur, tal como ya se ha propuesto en otras partes (Bidaseca et al., 2016; Santos, 2009).

Por lo tanto, el abordaje simultáneo supone delimitar un problema teórico que habilita a comparar perspectivas sin homogeneizarlas. En el análisis de esta simultaneidad, emergen dimensiones claves que permiten detectar dos cuestiones decisivas: a) un mismo problema que atraviesa a un grupo de autores de una determinada época o varias de ellas; b) la convergencia o contraposición entre distintas reflexiones respecto tanto de sus giros analíticos (de conceptos y de modalidades de elaborar teoría) como de ciertas tensiones que recorren a las perspectivas analizadas.

En primer lugar, la elección de un problema teórico para su investigación (aunque podría ser más de uno) supone demarcarlo mediante preguntas claves, de las cuales los autores estudiados dan cuenta y pretenden responder con sus elaboraciones. Esas preguntas teóricas y analíticas que recorren a las perspectivas seleccionadas tienen una encarnadura histórica teórica y epocal. Sobre el eje teórico, este implica comprender las discusiones y rupturas puestas en juego en un momento de una disciplina o de varias de ellas. Acerca de su eje epocal, esas discusiones se enmarcan en, y son parte de, encrucijadas sociales que, en determinado periodo, resultan más intensas que otras, por lo cual se despliegan de forma explícita o implícita en las obras analizadas. De esta manera, una investigación puede enfatizar uno de los dos ejes: detenerse en los presupuestos teóricos y epistemológicos de varias perspectivas; o pretender abarcar a ambos de forma combinada. Así, un problema teórico puede ser delimitado y estudiado desde la simultaneidad de perspectivas tanto del Sur como del Norte, para captar los aportes singulares de ambas, las diferentes modalidades de elaboración teórica y las preguntas compartidas.

Con base en estos dos ejes, el análisis de un problema teórico no se compone de unas definiciones más o menos ajustadas de tal problema, es decir, de un compendio al estilo de un manual. Tal análisis será el resultado de la confección de múltiples dimensiones que van emergiendo en el curso de la investigación comparativa. Desde esas dimensiones se plantea la posibilidad de profundizar en el carácter simultáneo de perspectivas del Sur y del Norte sin por ello esquematizarlas en búsqueda de una semejanza o divergencia radical, impuestas de modo forzado por la mirada del investigador, sin que sean el resultado de la propia investigación. Como ya he afirmado, esa simultaneidad es compleja, está llena de vericuetos, giros y tensiones.

En segundo lugar, ya sea que se focalice en una época determinada o en varias de ellas, este abordaje simultáneo analiza los modos a través de los cuales un con- 
junto de perspectivas elabora un cúmulo de preocupaciones teóricas. Al estudiar tales modos, se vuelven observables las rupturas y giros específicos que adoptan los enfoques sobre un mismo problema. Es allí, y no a partir del mero contraste entre conceptos, que se reconstruyen las convergencias o contraposiciones entre perspectivas. Tales convergencias o contraposiciones no son monolíticas; por el contrario, están atravesadas por tensiones. Una de estas resulta ser, justamente, la relación entre Sur y Norte, que implica una reflexión sobre los lazos de dominación entre estas regiones y dentro de ellas (el Sur en el Norte y el Norte en el Sur).

Esas tensiones Sur-Norte suponen una tematización de las formas de representación de tal dominación y de las formas de lucha frente a ella, de las cuales puede formar parte la misma teoría social o sociológica en sus diferentes contextos y posibilidades sociales e institucionales. De este modo, un abordaje simultáneo colabora con una interpretación de las relaciones entre el Sur y el Norte que no simplifica los complejos entramados entre relaciones de dominación, luchas por la emancipación y producción intelectual (en este caso, de la teoría sociológica). Al poner en simultáneo perspectivas de distintas regiones, aparecen las relaciones entre producciones del Sur y del Norte, sus temáticas, sus cruces posibles y sus más diversas reapropiaciones, que no responden a adscripciones lineales, ni institucionales ni teóricas. Así, el abordaje simultáneo desarma una mirada que se sustenta en la recepción (más allá de que a esa recepción se la complemente con la creatividad local), al enfocarse en el complejo entramado que implica la simultaneidad de una modernidad desigual. Se revelan, entonces, las afinidades y las convergencias, al igual que las diferencias singulares, las rupturas y las tensiones entre distintas regiones atravesadas por conflictos SurNorte entre ellas y dentro de ellas.

En definitiva, considero que un abordaje simultáneo de teorías sociológicas del Sur y del Norte permite analizar más fructíferamente tales perspectivas: se pueden estudiar y reelaborar unas y otras desde cada uno de los espacios sociales, para luego volver a reflexionar sobre las condiciones y cualidades de determinados enfoques. Esto es relevante en especial para dar cuenta de los aportes a la teoría sociológica que se han realizado — y se siguen realizando- desde el Sur, sin retrasarlos ni volverlos inconmensurables respecto de los que se realizan desde el Norte. Se puede fortalecer por esta vía el frágil pero creciente subcampo de la teoría sociológica argentina y latinoamericana, que debe hacer frente a las más diversas impugnaciones que van desde el empirismo hasta el relativismo cultural. La sociología requiere de construcciones e innovaciones teóricas que acompañen y sean parte clave de los análisis y diagnósticos de la modernidad, en especial, de una modernidad periférica o del Sur.

Al desafiar relaciones de jerarquías entre espacios de saber, en particular las asimétricas de elaboración y circulación de producciones teóricas, un abordaje 
simultáneo contribuye a una mayor reflexividad de la disciplina y sus presupuestos. La ciencia de la sociedad moderna requiere incorporar de forma decisiva los aportes y desafíos surgidos tanto en el Sur como en el Norte. Por eso reivindico la posibilidad de elaborar teoría sociológica en el Sur, desde la cual se pueda dialogar y ponerse en simultáneo con las miradas de otros espacios sociales, ya que esta posibilidad es fundamental para enfrentar y desmontar la reproducción de cierta división internacional del trabajo intelectual.

\section{Conclusiones}

En este artículo he desplegado una propuesta metodológica para estudiar comparativamente elaboraciones de la teoría sociológica del Sur y del Norte. He denominado a tal propuesta abordaje simultáneo, ya que implica colocar en un mismo plano temporal y analítico los aportes de distintos ámbitos regionales de producción de conocimientos y saberes. Para esto se requiere rastrear ciertos problemas teóricos y sus dimensiones emergentes, que se modulan y entrecruzan de distintas formas según las elaboraciones seleccionadas. Estas se encuentran marcadas por determinadas encrucijadas teóricas y epocales, las cuales atraviesan en simultáneo al Sur y al Norte en un momento de la modernidad — definida por su carácter conectado y desigual— en tanto forma relacional de comprender sus espacios y tiempos.

Con ello se pretende evitar las dicotomías que suponen al Norte como ámbito de elaboración teórica y al Sur como campo de recepción creativa o de una originalidad cultural incomensurable — más allá del signo positivo o negativo que se le otorgue a cada uno de los polos del dualismo-. Estas dicotomías se vinculan a los dispositivos de distanciamiento temporal modernos entre Sur y Norte que plasman distintos regímenes de historicidad y de escritura historiográfíca y sociológica: aquellos que alejan al Sur en el tiempo Histórico a partir de una representación única de la temporalidad desde el Norte, por ejemplo, con señas evolucionistas; y aquellos que separan de forma inconmensurable al tiempo del Sur, a modo de afirmación de una mirada relativista intraducible entre distintas temporalidades. Ambas posiciones requieren revisarse desde una perspectiva sustentada en un análisis de la simultaneidad compleja que despliegue para la teoría sociológica las conexiones entre Sur y Norte, sin describir sus retrasos o enfocarse solo en las recepciones originales, a la vez que evada simplificar la coetaneidad moderna en términos de presentismo e instantaneidad globalizada. De esta manera, a mi entender, las implicaciones teóricas de este abordaje son múltiples.

En el marco general de la teoría sociológica, no solo puede enriquecer sus elaboraciones con la inclusión de aportes decisivos producidos en el Sur, sino 
también habilitar a rastrear problemas teóricos y sus dimensiones que quedan poco explícitos o invisibilizados, debido a la falta de comparaciones de mayor calado que reivindiquen las contribuciones tanto del Sur (o periféricas) como del Norte (o centrales). Así también, respecto de la investigación en teoría sociológica desde el Sur y, en particular, desde Latinoamérica, este abordaje haría posible una mayor reflexividad del propio subcampo de conocimiento. Esto volvería más factible robustecer líneas de investigación singulares articuladas con debates trazados desde distintos espacios y tiempos conectados entre sí de forma compleja. Tales líneas de investigación se dirigirían a la continuidad de los esfuerzos actuales en teoría sociológica y a la recuperación de reflexiones pasadas con base en los análisis comparativos de obras del Sur y del Norte.

Asimismo, un abordaje simultáneo facilitaría una comprensión de la relación entre encrucijadas teóricas y epocales, ya que ese vínculo no puede analizarse sin establecer una mirada simultánea sobre las conexiones de la modernidad, que implican en, y frente a, encrucijadas epocales y modalidades específicas de elaboración teórica, de análisis de la modernidad y de pretensiones de intervención político-práctica. Esto lleva a reflexionar sobre las relaciones asimétricas entre Sur y Norte e incluso sobre la definición misma del Sur y del Norte en cuanto tales. En conclusión, el abordaje simultáneo pretende extender la investigación y el debate en teoría sociológica de modo tal que esta pueda conseguir una mayor profundidad analítica, para lo cual debe tener en cuenta y poner en cuestión las asimetrías en sus formas de producción y circulación al fortalecer su elaboración en el Sur y, en especial, en Latinoamérica.

\section{Referencias}

Alexander, J. (1982). Theoretical Logic in Sociology. Volume I: Positivism, Presuppositions, and Currents Controversies. California: University of California Press.

Aronson, P. (2011). De la crítica a la reconstrucción. Alternativas de las trayectorias sociológicas. Revista Pilquén, 14, 1-12.

Beigel, F. (2013). Centros y periferias en la circulación internacional del conocimiento. Nueva Sociedad, 245, 110-123.

Beigel, F. (2006). Vida, muerte y resurrección de las "teorías de la dependencia”. En AA. VV. Crítica y teoría en el pensamiento social latinoamericano (pp. 287-326). Buenos Aires: CLACso.

Bhambra, G. (2014). Connected sociologies. Londres: Bloomsbury Publishing. 
Bhambra, G. (2010). Sociology after Postcolonialism: Provincialized Cosmopolitanism and Connected Sociologies. En Boatcă, M., Costa, S. \& Gutiérrez-Rodríguez, E. (Comps.). Decolonizing European Sociology: Trans-disciplinary Approaches (pp. 33-47). Aldershot: Ashgate.

Bialakowsky, A. (2013). Antecedentes y posibilidades de un análisis comparativo en metateoría. El abordaje problemático en la teoría sociológica contemporánea. Documentos de Jóvenes Investigadores 38. Instituto Gino Germani.

Bidaseca, K. et al. (2016). Genealogías criticas de la colonialidad en América Latina, África, Oriente. Buenos Aires: CLACSO/IDAES.

Birth, K. (2008). The creation of coevalness and the danger of homochronism. Journal of the Royal Anthropological Institute, 14, 3-20.

Blanco, A. (2006). Razón y Modernidad. Gino Germani y la sociología en la Argentina. Buenos Aires: Siglo XXI.

Boatcă, M. \& Costa, S. (2010). La sociología poscolonial. Estado del arte y perspectivas. Estudios Sociológicos, XXVIII(83), 335-338.

Bourdieu, P. (2000). Las condiciones sociales de la circulación de las ideas. En Intelectuales, política y poder (pp. 159-170). Buenos Aires: Eudeba.

Burawoy, M. (2008). Rejoinder: For a Subaltern Global Sociology. Current Sociology, 56(3), 435-444.

Castro-Gómez, S. (2000). Ciencias sociales, violencia epistémica y el problema de la "invención del otro". En Lander, E. (Comp.). La colonialidad del saber: eurocentrismo y ciencias sociales. Perspectivas latinoamericanas (pp. 145-161). Buenos Aires: CLACso.

Chernilo, D. (2007). A Social Theory of the Nation-State: Beyond Methodological Nationalism. Londres: Routledge.

Cristiano, J. et al. (2015). Operaciones y procedimientos de construcción teórica: presentación preliminar de un instrumento de análisis. Primer Congreso Latinoamericano de Teoría Social, 19-21 de agosto. Buenos Aires, Argentina.

De Campos, H. (2000). De la razón antropofágica y otros ensayos. México: FCE.

De Marinis, P. (2013). Gemeinschaft, community, comunidad: algunas reflexiones preliminares acerca de las variadas semánticas de la comunidad en la teoría sociológica. Revista Argentina de Ciencia Política, (16), 87-104. 
De Marinis, P. (2012). Introducción: la comunidad en la teoría sociológica. En De Marinis, P. (Comp.). Comunidad: estudios de teoría sociológica (pp. 9-28). Buenos Aires: Prometeo Libros.

Domingues, J. M. (2009). La modernidad contemporánea en América Latina. Buenos Aires: CLACSO/Siglo XXI.

Dubey, M. (2002). Postmodern geographies of the U.S. South. Nepantla: Views from South, 3(2), 351-371.

Eisenstadt, S. (2013). América Latina y el problema de las múltiples modernidades. Revista Mexicana de Ciencias Políticas y Sociales, 58(218), 153-164.

Eisenstadt, S. (2000). Multiple modernities. Daedalus, 129(1), 1-29.

Fabian, J. (2002). Time and the Other. How anthropology makes its object. Nueva York: Columbia University Press.

Fanon, F. (2009). Piel negra, máscaras blancas. Madrid: Akal.

García, L. I. (2010). La crítica entre culturas. El problema de la "recepción" en el ensayo latinoamericano. Cuyo: Anuario de Filosofía Argentina y Americana, 27, 53-76.

Giddens, A. (1999). Consecuencias de la Modernidad. Madrid: Alianza.

Girola, L. (2015). ¿Qué hacemos cuando hacemos teoria? Una discusión acerca de aportaciones recientes y tres estudios de caso. Primer Congreso Latinoamericano de Teoría Social, 19-21 de agosto. Buenos Aires, Argentina.

Girola, L. \& Zabludovsky, G. (1991). La teoría sociológica en México en la década de los ochenta. Sociológica, 6(15), 11-63.

González, H. (2004). Retórica y locura. Para una teoría de la cultura Argentina. Buenos Aires: Colihue.

González Casanova, P. (1969). Sociología de la explotación. México: Siglo XXI Editores.

Grüner, E. (2010). La oscuridad y las luces. Capitalismo, cultura y revolución. Buenos Aires: Edhasa.

Habermas, J. (2000). La constelación posnacional. Buenos Aires: Paidós.

Hartog, F. (2014). El nombre y los conceptos de historia. Historia Crítica, 54, 75-87. 
Hartog. F. (2010). El historiador en un mundo presentista. En Devoto, F. (Comp.). Historiadores, ensayistas y gran público: la historiografia argentina, 1990-2010. Buenos Aires: Biblos.

Hartog, F. (2007). Regimenes de historicidad. Presentismo y experiencia en el tiempo. México: Universidad Iberoamericana.

Harvey, D. (1979). Urbanismo y desigualdad social. México: Siglo XXI.

Lander, E. (2000). Ciencias sociales: saberes coloniales y eurocéntricos. En Lander, E. (Comp.), La colonialidad del saber: eurocentismo y ciencias sociales. Perspectivas Latinoamericanas (pp. 11-40). Buenos Aires: CLACSO.

Lander, E. (1997). Modernidad, colonialidad y postmodernidad. Revista Venezolana de Economía y Ciencias Sociales, 3(4), 11-28.

Luhmann, N. (1997). Globalization or world society: How to conceive of modern society? International review of sociology, 7(1), 67-79.

Mascareño, A. (2008). Acción, estructura y emergencia en la teoría sociológica. Revista de Sociología, 22, 217-256.

Mudrovcic, M. (2013). Regímenes de historicidad y regímenes historiográficos: del pasado histórico al pasado presente. Historiografías: revista de historia y teoría, 5, 11-31.

Quijano, A. (2014). Colonialidad del poder y clasificación social. Cuestiones y horizontes. Antología esencial. Buenos Aires: ClACSO.

Offe, C. (2006). Autorretrato a distancia: Tocqueville, Weber y Adorno en los Estados Unidos de América. Buenos Aires: Katz.

Palti, E. (2014). Roberto Schwarz y el problema de "las ideas fuera de lugar". Aclaraciones necesarias y contradicciones cuarenta años después. Avatares Filosóficos, 1, 76-82.

Palti, E. (2004). Koselleck y la idea de Sattelzeit. Un debate sobre modernidad y temporalidad. Ayer, 53, 63-74.

Pascual, C. M. (2014). El giro espacial en historia. Derivas conceptuales y racconto historiográfico en Argentina. Imaginar los espacios de segregación localizados. Revista de Direito da Cidade, 6(2), 427-452.

Pereyra, D. (2010). Los científicos sociales como empresarios académicos. El caso de Gino Germani. En Pereyra, D. (Comp.), El desarrollo de las ciencias sociales. Tradiciones, actores e ins- 
tituciones en Argentina, Chile, México y Centroamérica (pp. 35-53). San José: Flacso Costa Rica.

Pignouli Ocampo, S. (2015). Una reformulación de la perspectiva sistemática en el marco de una sociología multiparadigmática. Primer Congreso Latinoamericano de Teoría Social, 19-21 de agosto. Buenos Aires, Argentina.

Rama, A. (1998). La ciudad letrada. Montevideo: Arca.

Requena Santos, F. (2000). Hacia una perspectiva reticular de la teoría sociológica. Papers. Revista de Sociología, (62), 133-144.

Ritzer, G. (1990). Metatheorizing in sociology. Sociological Forum, 5(1), 3-15.

Santos, B. de S. (2009). Una epistemología del sur: la reinvención del conocimiento y la emancipación social. México: Siglo XXI.

Santos, B. de S. (2003). Los procesos de globalización. En La caída del Ángelus Novus: ensayos para una nueva teoría social y una nueva práctica politica (pp. 167-242). Bogotá: ILSA.

Schrecker, C. (Ed.) (2012). Transatlantic voyages and sociology: The migration and development of ideas. Nueva York: Routledge.

Schwarz, R. (2014). Las ideas fuera de lugar. Meridional. Revista Chilena de Estudios Latinoamericanos, 3, 183-199.

Scribano, A. (2004). Conocimiento socialmente disponible y construcción de conocimiento sociológico desde América en Latina. Investigaciones Sociales, 8(12), 289-311.

Soja, E. (1989). Postmodern Geographies. The reassertion of space in Critical Social Theory. Londres: Verso.

Wallerstein, I. (2005). Análisis de sistemas-mundo: Una introducción. México: Siglo XXI.

Zabludovsky G. (2012). El debate conceptual y las "teorías de alcance intermedio": a propósito de la sociología en México. Acta Sociológica, 59, 103-133.

Zabludovsky, G. (2002). Teoría y metateoría en las ciencias sociales contemporáneas. En Sociología y política, el debate clásico y contemporáneo. Teoría y metateoría en las ciencias sociales contemporáneas (pp. 127-168). México: M. Á. Porrúa. 